\title{
Low physical functioning and impaired performance of activities of daily life in COVID-19 patients who survived hospitalisation
}

\author{
To the Editor:
}

Thousands of Italians with coronavirus disease 2019 (COVID-19) have been admitted to hospital. In the Piedmont Region, as of 12 May 2020, 12272 patients survived hospital admission [1], and most of them were discharged home. As the influx of COVID-19 patients is exceeding the hospital bed capacity in Northern Italy, patients are discharged home after two negative reverse transcription (RT)-PCR tests for the severe acute respiratory syndrome coronavirus 2 (SARS-CoV-2), notwithstanding their physical status. Nevertheless, lower-limb muscle deconditioning and an impaired performance of activities of daily living (ADLs) are likely to occur. Indeed, COVID-19 patients suffer from multiple symptoms during hospitalisation, and the acute care takes place in strict isolation, which will reduce a patient's mobility to zero [2].

To date, no data exist on physical functioning and performance of ADLs at discharge from hospital. Nevertheless, it seems reasonable to hypothesise that patients with COVID-19 are in need of rehabilitative interventions directly following hospital admission [3]. This retrospective study assessed the proportion of patients with low physical functioning and/or impaired performance of ADLs at the time they were discharged home.

In this study, the routine data of patients with COVID-19 admitted to rehabilitation institute Istituti Clinici Scientifici Maugeri, IRCCS (Veruno, Italy) were analysed. Normally, this is a specialised rehabilitation clinic, including a department for pulmonary rehabilitation. During the COVID-19 pandemic, the department for pulmonary rehabilitation was transformed into a COVID-19 ward, taking hospitalised patients with confirmed SARS-CoV-2 transferred from surrounding acute care hospitals who did not have enough bed capacity. Participants signed a general consent that allows future use of their medical records for medical research. Pre-existing data were de-identified prior to analyses.

During the hospitalisation in Veruno, patients received medical treatment for COVID-19 and early mobilisation/bedside physiotherapy (maximum $30 \mathrm{~min} \cdot \mathrm{day}^{-1}$ ); some also received psychological counselling. This was not a formal comprehensive interdisciplinary pulmonary rehabilitation programme as described previously [4]. At the time of transfer from the acute care hospital to Veruno, and at discharge home (i.e. two negative RT-PCR tests for SARS-CoV-2), patients performed several routine clinical tests: the 1-min sit-to-stand (STS) test was performed as a measure of physical fitness [5]; the Short Physical Performance Battery (SPPB) was used to assess lower extremity function [6]; and the Barthel index was used to assess performance in ADLs ( $\leqslant 60$ points indicates severe dependency) [7]. Finally, the patient's sex, age, body mass index (BMI), comorbidities (all comorbidities with a severity score of 3-5 (moderate to very severe) on the cumulative illness rating scale [8]), and length of stay were recorded.

Mean \pm SD and proportions were used for descriptive statistics, as appropriate. Changes over time were analysed with a paired t-test. Moreover, Pearson's correlations coefficients were calculated between age,

@ERSpublications

About half of post-COVID-19 patients had severe impairments in physical functioning and during activities of daily living at discharge home, providing a clear rationale to study the safety and efficacy of rehabilitative interventions in these patients https://bit.ly/3hJvXJa

Cite this article as: Belli S, Balbi B, Prince I, et al. Low physical functioning and impaired performance of activities of daily life in COVID-19 patients who survived hospitalisation. Eur Respir J 2020; 56: 2002096 [https://doi.org/10.1183/13993003.02096-2020]. 
BMI and length of stay with 1-min chair stands, SPPB summary score and Barthel index at discharge home. The level of significance was set at $\mathrm{p}<0.01$.

Data from the first 115 consecutive patients (49.5\% women, age $74.3 \pm 12.7$ years, BMI $26.2 \pm 5.7 \mathrm{~kg} \cdot \mathrm{m}^{-2}$ ) with RT-PCR-confirmed SARS-CoV-2 infection were analysed. These patients were transferred to Veruno after a mean \pm SD $15.6 \pm 10.6$ days in the acute care hospital. During their stay in Veruno, four patients were sent to the emergency department for non-COVID-19-related medical emergencies, and eight patients died 2-45 days after the transfer. These eight patients (62.5\% women) were elderly (age $83.3 \pm 5.8$ years), with a high number of comorbidities $(5.5 \pm 2.1,87.5 \%$ had $\geqslant 4$ comorbidities), and $87.5 \%$ were bedridden at the time of entry in Veruno.

In total, 103 patients ( $51.5 \%$ men, age $73.9 \pm 12.9$ years, BMI $26.6 \pm 5.8 \mathrm{~kg} \cdot \mathrm{m}^{-2}$ ) survived the hospitalisation and were discharged home after a mean \pm SD stay in Veruno of $16.1 \pm 7.4$ days. 82 patients $(79.6 \%)$ did not receive ventilatory support in the acute care hospital, nine patients $(8.7 \%)$ received noninvasive ventilation (NIV), while 12 patients (11.7\%) received mechanical ventilation and NIV. The mechanically ventilated group stayed significantly longer in the acute care hospital compared to the remaining group of patients (33.0 \pm 12.1 versus $16.8 \pm 7.6$ days; $\mathrm{p}<0.001)$. In contrast, the length of stay in Veruno was shorter for the mechanically ventilated group: $11.1 \pm 3.4$ versus $16.8 \pm 7.6$ days $(\mathrm{p}=0.012)$.

For the 103 patients who were discharged home, the mean \pm SD number of comorbidities was $3.8 \pm 2.0,88 \%$ had $\geqslant 2$ comorbidities, and $55 \%$ had $\geqslant 4$. Many patients had dyspnoea/cough/fever (90\%), depression (33\%), dysphagia (16\%) and/or cognitive impairment (33\%). Moreover, $78.6 \%$ of the patients had oxygen supplementation (inspiratory oxygen fraction $0.24-0.45$ ).

At entry in Veruno, only $42 \%$ of the patients were able to do the 1-min STS test. The mean \pm SD number of 1-min chair stands was $14 \pm 6$ repetitions, which was for $74.4 \%$ of the patients below percentile 2.5 of the reference values [9]. All patients performed the SPPB, which showed that $78.6 \%$ of the patients were in the low-performance group (0-6 points), $13.5 \%$ of the patients were in the moderate-performance group (7-9 points), and $7.8 \%$ of the patients were in the high-performance group (10-12 points) [10]. 67\% of the patients scored poorly ( $\leqslant 60$ points) on the Barthel index, and $45.6 \%$ of the patients were bedridden. After stratification for mechanical ventilation in the acute care hospital (yes versus no), the proportion of patients with a low SPPB summary score $(83.3 \%$ versus $78.0 \%$, respectively) or with a poor Barthel index score $(66.7 \%$ versus $67.0 \%$, respectively) was comparable between groups.

At discharge home, mean (95\% CI) scores were significantly better for the number of 1-min chair stands (6.2 (4.7-7.7); $\mathrm{p}<0.001)$, the SPPB summary score $(2.1(1.6-2.6)$ points; $\mathrm{p}<0.001)$ and the Barthel index (15 (11-19) points; $\mathrm{p}<0.001)$. However, $33.3 \%$ of the patients still had a number of STS repetitions in $1 \mathrm{~min}$ that was below percentile 2.5 of the reference values [9]. Moreover, the majority of patients had low (53.3\%) or moderate (17.5\%) SPPB summary scores, and $47.5 \%$ of the patients still scored poorly on the Barthel index (figure 1). Problems were still reported for feeding ( $7 \%$ unable, $17 \%$ needed help), personal toileting (35\% needed help), bathing (64\% dependent), dressing/undressing ( $24 \%$ dependent, $28 \%$ needed help), toilet use (35\% dependent, $13 \%$ needed some help), controlling bladder ( $25 \%$ incontinent, $10 \%$ occasional accident), controlling bowel (21\% incontinent, $14 \%$ occasional accident), transfer (15\% unable, no sitting balance; $29 \%$ needed major help, can sit), mobility (35\% immobile; $30 \%$ wheelchair independent, including corners; 30\% walking with help), and ascending/descending stairs (65\% unable,

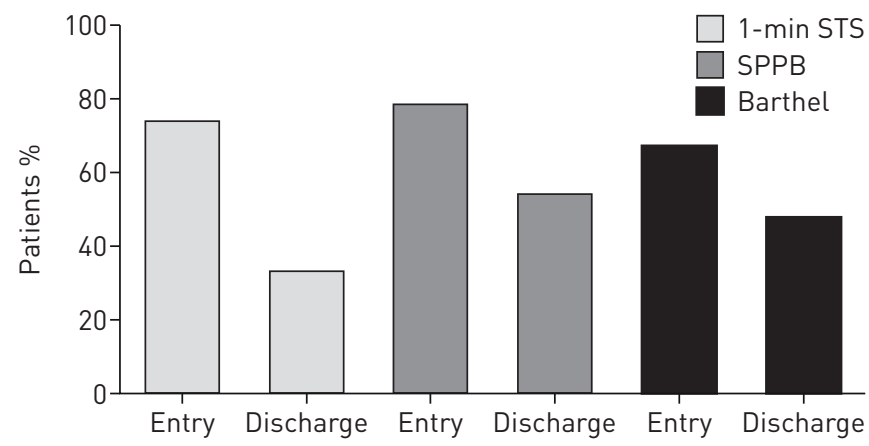

FIGURE 1 Proportions of patients with abnormal physical functioning and/or abnormal performance of activities of daily living: patients (at entry in Veruno and at discharge home) with an abnormally low number of 1-min chair stands in the 1-min sit-to-stand (STS) test (below percentile 2.5 of the reference values [9]), with a summary score on the Short Physical Performance Battery (SPPB) of 0-6 points (low performance), or with a poor score ( $\leqslant 60$ points) on the Barthel index. 
$18 \%$ needed help). Finally, $17.5 \%$ of the patients were still bedridden, and $21 \%$ of the patients had oxygen supplementation at discharge. Age, BMI and length of stay were not associated with number of 1-min chair stands, SPPB summary score and Barthel index at discharge home (all p>0.10).

This is the first study to report low physical functioning and impaired performance of ADLs in COVID-19 patients who survived hospitalisation. Therefore, it seems fair to conclude that COVID-19 patients discharged home after hospitalisation, in acute and subsequent post-acute wards, have a clear need for rehabilitative interventions. Indeed, initial recovery under the treatment/supervision of the inpatient physiotherapy team was noticed during the post-acute hospitalisation. However, physical functioning and performance of ADLs were still significantly hampered at discharge home. The current results resemble findings in patients with COPD, who are weakened, have low physical functioning and poor performance of ADLs at discharge home following an exacerbation-related hospitalisation [10,11]. Interestingly, early post-hospitalisation pulmonary rehabilitation has been shown to be safe and beneficial (e.g. resulting in better physical functioning and quality of life) in patients with COPD [12]. Timely referral to post-hospitalisation rehabilitative interventions in Northern Italy seems very difficult, as present accessibility is limited. In fact, many specialised pulmonary rehabilitation facilities are currently used for COVID-19 care, like the one in Veruno. Moreover, the number of post-COVID-19 patients in need of rehabilitation is likely to outnumber the regular capacity of specialised pulmonary rehabilitation clinics. Finally, a lockdown does not seem to be an encouraging environment to become physically active [13]. Therefore, it seems reasonable to hypothesise that these elderly, multimorbid post-COVID-19 patients will have a slow and/or incomplete post-hospitalisation recovery. This may have detrimental consequences for the patients' autonomy and participation, and cause both caregiver burden for family and friends and increased healthcare resource utilisation.

Interestingly, only 12 of the 103 patients were mechanically ventilated in the acute care hospital, which suggests that hospitalised COVID-19 patients without mechanical ventilation merit post-hospital follow-up. The retrospective study did not capture any details of physical functioning before hospitalisation, which may already have been impaired. Also, patients' emotional and social status are unknown, but deserve adequate diagnostics and treatment. Indeed, the post-COVID-19 patients with multiple physical, emotional and social treatable traits may be excellent candidates for a comprehensive pulmonary rehabilitation programme, including also occupational therapy and dietary interventions [14].

The current sample of patients with confirmed COVID-19 seems representative for the hospitalised patients in Italy. Then again, the current sample seems older in age and to have a lower mean BMI than hospitalised COVID-19 patients treated in the USA [15].

To conclude, post-COVID-19 patients can have impaired physical functioning when they are discharged home, even after early mobilisation/bedside physiotherapy. These data suggest that early referral to rehabilitative intervention options in the post-hospitalisation phase should be considered.

Stefano Belli $\oplus^{1}$, Bruno Balbi ${ }^{1}$, Ilaria Prince ${ }^{1}$, Davide Cattaneo $\odot^{1}$, Francesca Masocco ${ }^{1}$, Sergio Zaccaria $^{1}$, Luca Bertalli ${ }^{1}$, Francesco Cattini ${ }^{1}$, Arianna Lomazzo ${ }^{1}$, Francesca Dal Negro ${ }^{1}$, Marica Giardini ${ }^{1}$, Frits M.E. Franssen $\oplus^{2,3,4}$, Daisy J.A. Janssen $\oplus^{2,5}$ and Martijn A. Spruit $\oplus^{2,3,4,6}$

${ }^{1}$ Pulmonary Rehabilitation of the Institute of Veruno, Istituti Clinici Scientifici Maugeri IRCCS, Novara, Italy. ${ }^{2}$ Dept of Research and Development, CIRO+, Horn, The Netherlands. ${ }^{3}$ Dept of Respiratory Medicine, Maastricht University Medical Center (MUMC+), Maastricht, The Netherlands. ${ }^{4}$ NUTRIM School of Nutrition and Translational Research in Metabolism, Maastricht, The Netherlands. ${ }^{5}$ Dept of Health Services Research, Care and Public Health Research Institute, Faculty of Health Medicine and Life Sciences, Maastricht University, Maastricht, The Netherlands. ${ }^{6}$ REVAL Rehabilitation Research Center, BIOMED Biomedical Research Institute, Faculty of Rehabilitation Sciences, Hasselt University, Diepenbeek, Belgium.

Correspondence: Stefano Belli, IRCCS Istituti Clinici Scientifici Maugeri SpA SB, Via Salvatore Maugeri 4, 27100 Pavia, Italy. E-mail: stefano.belli@icsmaugeri.it

Received: 1 June 2020 | Accepted after revision: 23 July 2020

Conflict of interest: S. Belli has nothing to disclose. B. Balbi has nothing to disclose. I. Prince has nothing to disclose. D. Cattaneo has nothing to disclose. F. Masocco has nothing to disclose. S. Zaccaria has nothing to disclose. L. Bertalli has nothing to disclose. F. Cattini has nothing to disclose. A. Lomazzo has nothing to disclose. F. Dal Negro has nothing to disclose. M. Giardini has nothing to disclose. F.M.E. Franssen reports grants and personal fees from AstraZeneca and Novartis, and personal fees from Boehringer Ingelheim, Chiesi, GlaxoSmithKline and TEVA, outside the submitted work. D.J.A. Janssen reports personal fees for lectures from Astra Zeneca, Novartis and Boehringer Ingelheim, outside the submitted work. M.A. Spruit reports grants from the Netherlands Lung Foundation and Stichting Astma Bestrijding, and grants and personal fees from AstraZeneca and Boehringer Ingelheim, outside the submitted work.

Support statement: This work was supported by the Ricerca Corrente Funding scheme of the Ministry of Health, Italy. Funding information for this article has been deposited with the Crossref Funder Registry. 


\section{References}

1 Ministero della Salute. Nuovo coronavirus [Novel coronavirus]. www.salute.gov.it/portale/nuovocoronavirus/ homeNuovoCoronavirus.jsp

2 Steardo L, Steardo L Jr, Zorec R, et al. Neuroinfection may contribute to pathophysiology and clinical manifestations of COVID-19. Acta Physiol 2020; 229: e13473.

3 European Respiratory Society. COVID-19 and rehabilitation. www.ersnet.org/covid-19-blog/covid-19-andrehabilitation Date last updated: 3 April 2020.

4 Spruit MA, Singh SJ, Garvey C, et al. An official American Thoracic Society/European Respiratory Society statement: key concepts and advances in pulmonary rehabilitation. Am J Respir Crit Care Med 2013; 188: e13-e64.

5 Bohannon RW, Crouch R. 1-Minute sit-to-stand test: systematic review of procedures, performance, and clinimetric properties. J Cardiopulm Rehabil Prev 2019; 39: 2-8.

6 Guralnik JM, Simonsick EM, Ferrucci L, et al. A short physical performance battery assessing lower extremity function: association with self-reported disability and prediction of mortality and nursing home admission. J Gerontol 1994; 49: M85-M94.

7 Mahoney FI, Barthel DW. Functional evaluation: the Barthel index. A simple index of independence useful in scoring improvement in the rehabilitation of the chronically ill. Md State Med J 1965; 14: 61-65.

8 Salvi F, Miller MD, Grilli A, et al. A manual of guidelines to score the modified cumulative illness rating scale and its validation in acute hospitalized elderly patients. J Am Geriatr Soc 2008; 56: 1926-1931.

9 Strassmann A, Steurer-Stey C, Dalla Lana K, et al. Population-based reference values for the 1-min sit-to-stand test. Int J Public Health 2013; 58: 949-953.

10 Spruit MA, Gosselink R, Troosters T, et al. Muscle force during an acute exacerbation in hospitalised patients with COPD and its relationship with CXCL8 and IGF-I. Thorax 2003; 58: 752-756.

11 Martínez-Velilla N, Valenzuela PL, Zambom-Ferraresi F, et al. Tailored exercise is safe and beneficial for acutely hospitalised older adults with chronic obstructive pulmonary disease. Eur Respir J 2020; in press [https://doi.org/ 10.1183/13993003.01048-2020].

12 Puhan MA, Gimeno-Santos E, Cates CJ, et al. Pulmonary rehabilitation following exacerbations of chronic obstructive pulmonary disease. Cochrane Database Syst Rev 2016; 12: CD005305.

13 Guralnik JM, Ferrucci L, Simonsick EM, et al. Lower-extremity function in persons over the age of 70 years as a predictor of subsequent disability. N Engl J Med 1995; 332: 556-561.

14 Pietrobelli A, Pecoraro L, Ferruzzi A, et al. Effects of COVID-19 lockdown on lifestyle behaviors in children with obesity living in Verona, Italy: a longitudinal study. Obesity 2020; 28: 1382-1385.

15 Argenziano MG, Bruce SL, Slater CL, et al. Characterization and clinical course of 1000 patients with coronavirus disease 2019 in New York: retrospective case series. BMJ 2020; 369: m1996. 